\title{
Nordiques
}

39 | 2020

Varia

\section{Erik Poppe, Utøya 22 juillet}

Film norvégien, 2018

Harri Veivo

\section{(2) OpenEdition}

\section{Journals}

Édition électronique

URL : http://journals.openedition.org/nordiques/617

DOI : $10.4000 /$ nordiques.617

ISSN : 2777-8479

Éditeur :

Association Norden, Bibliothèque de Caen la mer

Référence électronique

Harri Veivo, "Erik Poppe, Utøya 22 juillet », Nordiques [En ligne], 39 | 2020, mis en ligne le 01 novembre 2020, consulté le 13 mars 2021. URL : http://journals.openedition.org/nordiques/617 ; DOI : https:// doi.org/10.4000/nordiques.617

Ce document a été généré automatiquement le 13 mars 2021

Nordiques 


\title{
Erik Poppe, Utøya 22 juillet
}

\author{
Film norvégien, 2018
}

\section{Harri Veivo}

\section{RÉFÉRENCE}

Erik Poppe, Utøya 22 juillet, film norvégien, 2018

1 Le spectateur qui entrait dans une salle de cinéma en 2019 pour voir Utøya 22 juillet ne savait pas forcément ce qui l'attendait. Le film de Erik Poppe est un objet hybride : œuvre d'art, participation à une discussion sociétale, travail pour surmonter un traumatisme collectif, et message politique. Il s'adresse à un public international et fut en effet sélectionné au festival de Berlin. En même temps, il est inextricablement lié au contexte norvégien. Réussir sur tous ces fronts est un exercice difficile. Utøya 22 juillet, pourtant qualifié de chef-d'œuvre en Norvège ${ }^{1}$, n'arrive pas à atteindre son but, mais son échec donne matière à réflexion.

2 Un film de 93 minutes montré dans une salle de cinéma, aussi réel et terrible que soit son sujet, ne peut pas échapper à son statut d'objet poétique, si l'on entend " poétique » dans le sens étymologique, dérivé de poiésis, c'est-à-dire relevant d'un travail de création qui implique des choix. Il faut regarder Utøya 22 juillet d'abord comme un film, comme n'importe quel film, même s'il est également autre. Pour raconter l'acte terroriste meurtrier - 69 victimes - perpétué par Anders Behring Breivik contre le camp d'été de la Ligue des jeunes travaillistes (Arbeidernes ungdomsfylking) le 22 juillet 2011, Poppe a créé une narration qui met le spectateur à la place des victimes. La première séquence montre, à l'aide de matériel documentaire provenant pour partie des caméras de surveillance, l'attentat à la bombe dans le quartier administratif du centre-ville d'Oslo qui a précédé la tuerie d'Utøya et causé la mort de 8 personnes. La violence est captée dans un clip qui montre l'effet de blast dans ce qui semble être une supérette, mais le regard reste éloigné. Commence alors un long plan-séquence qui ramène le spectateur sur l'île et parmi les victimes. On découvre un groupe de jeunes à travers un champ de vision et une bande sonore qui créent une illusion de 
participation. Dans la première scène, le personnage central du film, Kaja (Andrea Berntzen), une jeune femme de 19 ans, regarde directement le spectateur et lui parle. Nous la suivrons ensuite jusqu'à la fin du film, courant, fuyant, se cachant désespérément, à travers une caméra qui adopte intégralement la position d'un personnage de l'histoire. La séquence dure le temps de l'attentat, 72 minutes, et le nombre de tirs est, lui aussi, le même que dans la réalité.

3 À partir de ces choix fondamentaux, le film construit un récit visuel rythmé : les moments où l'on fuit alternent avec les moments où l'on reste caché, de même que les moments de solitude alternent avec les moments où l'on est ensemble; quelques plans rapprochés sur des détails et vues plus ouverts sur l'espace offrent un contrepoids au champ de vision restreint au point de vue d'un personnage. La narration réussit à maintenir une dynamique efficace: l'action et l'inertie s'alternent, les dialogues succèdent à des silences interrompus uniquement par les tirs. Le récit montre comment les jeunes basculent de l'incrédulité et du déni au désespoir. Les éléments sont savamment organisés : le groupe initial est dispersé, puis les jeunes se retrouvent à deux ou à trois dans des scènes qui permettent de verbaliser le vécu et réaffirmer les liens qui les unissent. Le thème qui domine - à part la terreur et la souffrance des victimes - est la série des choix éthiques entre différentes formes de loyauté que Kaja doit assumer : leader naturel, elle a la capacité d'aider, diriger et consoler les autres, mais elle se sent aussi responsable de sa petite sœur Emilie (Elli Rhiannon Müller Osbourne) qui reste introuvable dans le chaos. Malgré son impossibilité à répondre à toutes les sollicitations, ses choix témoignent de la compassion et de la solidarité. On dit facilement que les situations d'exception montrent la vérité de l'être humain qui serait son égoïsme fondamental. Ce n'est pas vrai : confronté à l'extrême, il est aussi capable de l'altruisme. Les récits de survivants sur lesquels le film se fonde le prouvent amplement ${ }^{2}$.

4 La première réplique du film est « Vous n'allez jamais comprendre ». Kaja le prononce en regardant le spectateur droit dans les yeux. Ensuite, elle se détourne de la caméra et l'on comprend qu'elle est train de parler à ses parents. Ce moment initial de contact qui entame le récit sur le massacre est essentiel pour saisir comment le film participe à la discussion sociétale sur l'attentat en Norvège. Dans le regard de Kaja, il y a une sollicitation à maintenir la relation qui vient d'être créée, de suivre la jeune femme, d'entrer dans son monde; et dans ses paroles, il y a un constat d'une distance infranchissable, d'une incapacité fondamentale de comprendre. En donnant ainsi la voix aux victimes et en construisant entre elles et le spectateur une relation qui repose sur l'impossible crête entre participation et extériorité, le film de Poppe présente une contribution essentielle à la discussion norvégienne sur l'événement. Selon Sindre Bangstad, les médias et la recherche ont placé le terroriste au centre des récits qu'ils ont constitués sur l'attentat, essayant d'expliquer l'inexplicable par la mégalomanie et le narcissisme de Breivik; les victimes et les survivants ont été destitués du droit sur la narration qui les concerne cependant en premier lieu, alors que certains d'entre eux reçoivent toujours des menaces sur les réseaux sociaux à cause de leur engagement politique ${ }^{3}$. Utøya 22 juillet plonge le spectateur dans la durée de l'événement, dans le désarroi et la peur, « comme si » l'on y était, tout en soulignant la distance qui sépare le public et les acteurs de l'expérience des victimes. Prétendre reconstituer le vécu des victimes serait indigne, tant la violence dont elles ont été l'objet reste incompréhensible à ceux qui ne l'ont pas éprouvé (et aux victimes elles- 
mêmes aussi, mais d'une manière fondamentalement autre). Pourtant, il faut que leurs récits puissent exister et que leurs voix se faire entendre, et c'est le cas dans ce film.

Un traumatisme collectif est causé par un événement qui menace l'existence du groupe et qui dépasse ses moyens de compréhension cognitive et émotionnelle. Le traumatisme résiste à l'explication. Il nécessite un travail d'interprétation qui s'inscrit dans la longue durée pour être intégré dans le réseau de significations, de récits et de valeurs qui former la communauté. Le double attentat du 22 juillet 2011 a constitué un tel événement pour la société norvégienne. Dans le film, l'expérience de la terreur, vécue individuellement, est reliée aux institutions qui sont vitales pour la communauté. Le terroriste s'était déguisé en policier, ce qui crée, chez les victimes, quand ils croient être chassés par les forces de l'ordre, un effondrement de la foi en l'institution même qui est censée garantir leur intégrité. Impitoyablement, le film montre aussi l'incapacité de l'autre institution essentielle, la famille, d'assurer la sécurité. Les parents que les jeunes appellent avec leurs portables ne répondent pas ou ne saisissent pas ce qui arrive à leurs enfants. L'ubiquité des liens familiaux créée par les téléphones portables et l'inutilité de ces mêmes liens virtuels face aux menaces réelles est d'ailleurs un élément qui caractérise l'expérience de la violence et de la sécurité aujourd'hui.

6 En montrant comment la violence casse le lien entre l'individu et la société et laisse les jeunes seuls face au terroriste, le film de Poppe confronte le spectateur avec l'expérience traumatisante en train de se produire et participe ainsi d'une manière singulière au travail commun pour la surmonter. Plusieurs auteurs ont déjà choisi de traiter le sujet, mais souvent d'une manière oblique, à travers des personnages et à l'aide de techniques qui maintiennent une distance vis-à-vis des victimes. C'est le cas par exemple du roman Velkommen til oss (2014) de Eivind Hofstad Evjemo qui décrit les effets de l'attentat du point de vue des voisins d'une famille qui a perdu un enfant; de même, la série 22. Juli (2020) de Sara Johnsen et Pål Sletaune aborde le drame d'Utøya à travers des personnages qui sont confrontés à l'événement du fait de leur profession, et non pas comme victimes. Le film Rekonstruktion Utøya (2019) du suédois Carl Javér offre bien la voix aux survivants, mais il le fait par le biais d'une reconstruction de l'événement a posteriori et non pas dans le présent de l'action comme dans le film de Poppe.

7 La difficulté du travail sur le trauma collectif s'est révélée au grand jour quand a éclaté la controverse autour du mémorial de l'attentat. La commission en charge du projet voulait une œuvre, qui « recréerait le trauma et les valeurs auxquelles [les Norvégiens] croient » au lieu de "figer le temps »5. Le mémorial était censé confronter la société avec l'attentat et l'engager dans une négociation sur l'identité et l'avenir du pays. Le choix initial du lieu - non pas sur l'île que le parti voulait continuer à utiliser pour les stages, mais dans une commune voisine non directement affectée par l'événement - a cependant été perçu comme un déplacement du trauma hors de son périmètre réel. Les détracteurs du projet ont ressenti que le mémorial chercherait à compenser le manque d'attachement territorial par une simulation esthétique douteuse. Ils ont exprimé également leur inquiétude de voir la commune devenir une destination de tourisme de la terreur suite à une intrusion du pouvoir étatique (le projet de mémorial national porté par l'État) au niveau local. La controverse s'est alimentée d'arguments géographiques, esthétiques, économiques et poétiques, montrant ainsi non seulement comment le travail sur un trauma collectif engage toute la société, mais aussi comment 
il évolue dans le temps. Le projet a été finalement abandonné, l'esprit de solidarité nationale que l'attentat avait créé se révélant insuffisant pour surmonter ces désaccords ${ }^{6}$. Par contre, le sort du café de l'île où plusieurs victimes ont été tuées a été négocié avec succès. La décision initiale de démanteler le bâtiment, devenu porteur de l'horreur de l'attentat, a été perçue par certains parents comme l'effacement du dernier lien qui leur restait à leur enfant disparu. La discussion a abouti à une modification du projet initial: aujourd'hui, le café est préservé et intégré à l'architecture du nouveau bâtiment construit au même emplacement ${ }^{7}$. Un épisode dans le film représente le désarroi et la panique des jeunes piégés dans le café.

Un aspect important du traitement du trauma par le film est la reconnaissance de l'existence d'une violence avant même que l'attentat n'ait lieu. Les Norvégiens et les habitants des pays nordiques en général se laissent facilement bercer par la présupposition d'un exceptionnalisme nordique : le mal qui existe ailleurs ne pourrait pas avoir lieu «chez nous ». Corollairement, ce que les Nordiques font hors de leur sol ne pourrait pas avoir de conséquences dans leurs propres pays, comme si le monde globalisé ne fonctionnait qu'en sens unique. La Norvège a cependant une longue histoire de terrorisme et elle a été impliquée dans des guerres sur le sol étranger ${ }^{8}$. Les jeunes du film semblent ignorer le premier fait, mais ils sont conscients du second. Lorsqu'ils apprennent qu'un attentat a eu lieu à Oslo, la participation de la Norvège à la FIAS en Afghanistan et l'Al-Qaïda sont les premiers éléments qu'ils évoquent pour tenter de comprendre l'événement.

9 La vraie nature de l'engagement de leur pays au Moyen-Orient - les jeunes ne sont pas d'accord s'il s'agit de guerre ou de maintien de paix - est un des rares contextes politiques que le film de Poppe évoque explicitement. En participant au travail sur le trauma collectif qui a profondément ébranlé les fondements même de la société norvégienne, Utøya 22 juillet ne peut cependant pas occulter une dimension politique plus fondamentale. "La politique apparaît dans l'espace entre-les hommes ${ }^{9}$ ", comme l'écrit Hannah Arendt, et le medium cinématographique se situe en effet littéralement entre les membres d'une communauté. Le film revendique cette dimension en insérant après le générique de fin un court texte qui affirme que «l'extrême droite connait aujourd'hui une poussée en Europe et dans l'ensemble du monde occidental. L'image de l'ennemi à abattre [dans l'original : The terrorist's concept of who the enemy is] est plus que jamais vivace et continuer à se répandre. » Plus loin, un autre texte précise qu'à la base du film réside "une vérité », mais que "d'autres peuvent exister». Le film prétend ainsi dénoncer un danger politique grandissant et admettre l'existence d'une multitude de perceptions véridiques du sujet dont il traite, tout en choisissant de n'en défendre qu'une seule. On peut se demander si cette équation est possible.

10 La micro-société des jeunes d'Utøya, telle que le film la construit, regroupe des profils variés : il y a Kaja le leader naturel qui rêve de carrière politique; sa sœur Emilie, une adolescente insouciante et boudeuse; Magnus (Alexander Holmen) qui est venu au camp pour draguer, mais qui se considère comme un loser ; Issa (Sarosh Sadat) le jeune réfugié d'origine afghane qui, grâce à son expérience des conflits armés, reconnaît les tirs alors que les autres croient entendre des pétards ; et d'autres encore, jeunes adultes et enfants, Norvégiens « ethniques »- une catégorie utilisée par le film pour qualifier Breivik - et autres ; une diversité. La tolérance est la valeur qui réunit ces personnes. Elle est affirmée par une banderole contre le racisme qui apparait dans une scène, une des occasions où le film prend ouvertement position. Les jeunes du film constituent une 
microsociété où les différences éthiques ou confessionnelles sont assimilées dans un cadre fédérateur laïque et typiquement norvégien qui tend à les effacer de la sphère publique. Il n'y a aucune référence à une religion dans le film. La diversité devient ici même un facteur de résilience quand Issa, grâce son expérience en Afghanistan, comprend le premier la gravité de la situation.

11 En même temps, Poppe a choisi de ne rendre présent le terroriste dans son film que par le son des tirs et trois scènes brèves où on le voit sous la forme d'une figure fantomatique sans traits personnels. Le film ne dit rien de ses motivations, alors que le manifeste qu'il a mis en ligne avant son passage à l'acte, intitulé 2083 - A European Declaration of Independence (publié sous le nom anglicisé Andrew Brewick), montre qu'il s'était doté de la tâche de lutter par le terrorisme contre ce qu'il percevait être l'islamisation de l'Europe. The terrorist's concept of who the enemy is est un amalgame délirant construit à partir de vraies et de fausses donnés statistiques sur l'immigration et l'évolution démographique des sociétés occidentales, des théories complotistes, des récits historiques instrumentalisés, et des menaces purement imaginaires. On ne voit rien de cela dans Utøya 22 juillet. Le terrorisme est sans visage, sans pensées et sans histoire, comme repoussé hors de la société.

En construisant une image en noir et blanc qui confronte une jeunesse exemplaire à un tueur anonyme, le film occulte une question essentielle : comment le repli identitaire typique à l'extrême droite nourrit la haine de la société tolérante, égalitaire et ouverte? Utøya 22 juillet ne permet pas d'appréhender la logique, l'imaginaire, la politique, les valeurs, la vision de la société et les autres facteurs sociaux ou individuels qui nourrissent ce processus meurtrier. De plus, il nous montre la menace de la violence d'extrême droite - et du terrorisme en général - comme quelque chose de radicalement étranger et incompréhensible, alors qu'il s'agit de personnes qui vivent parmi nous, qui sont semblables à nous. Dans sa promotion d'une identité norvégienne et occidentale pensée en termes essentialistes et fondée sur une version sécularisée du Christianisme qui serait inévitablement opposé à un Islam prétendument inconciliable avec la démocratie et l'égalité, le manifeste de Breivik s'apparente en fait à la politique du parti d'extrême droite norvégien Fremskrittspartiet (dont il a été membre pendant plusieurs années) et de ses homologues nordiques et européens, comme Frédérique Harry l'a noté ${ }^{10}$. Le combat politique et éthique pour une meilleure société ne se joue pas entre les deux pôles extrêmes que le film de Poppe montre, mais dans la zone grise de notre vie quotidienne.

13 Les choix poétiques du film sont réussis en termes cinématographiques. Il apporte une importante contribution au travail collectif sur le trauma. Ce qui est gagné dans ces domaines est perdu ailleurs. Utøya 22 juillet oublie la vérité qui est peut-être la plus difficile à supporter et échoue ainsi à délivrer un message politique convaincant et utile. 


\section{NOTES}

1. Birger Vestmo, «Utøya 22 juli. Intet mindre en ett mesterverk », Filmpolitiet 18 mars 2018, en ligne à https://p3.no/filmpolitiet/2018/02/utoya-22-juli/. Consulté le 20 mai 2020.

2. Ces récits se trouvent aussi dans En av oss. En fortelling om Norge de Åsne Seierstad, une narration et analyse de la vie de Breivik et de trois victimes. Åsne Seierstad, En av oss. En fortelling om Norge de, Oslo, Kagge, 2013.

3. Sindre Bangstad, «Don't look now. Can Norway reckon with the reality of right-wing extremists?", World Policy Journal, vol. 35, n², 2018, p.34-40. Sur les menaces, voir « De overlevde Utøya ", Aftenposten, 19 juillet 2018 ; «Tarjei, 26, sköts på Utøya - nu dödshotas han », Expressen, 22 juillet 2018.

4. Trad. française. Vous n'êtes pas venus au monde pour rester seuls, trad. par Terje Sinding, Paris, Grasset, 2017.

5. Jørn Mortensen, conseiller artistique du concours sur le mémorial, cité dans Charlotte HathKelly, "Memory Wound: architectural controversies in Norway after the July 22 attacks ", Ethnologie française, $\mathrm{n}^{\circ}$ 179, 1/2019, p. 122. Disponible en ligne à https://www.cairn.info/revueethnologie-francaise-2019-1-page-119.htm. Consulté le 7 février 2019.

6. Sur l'évolution du climat d'opinion, voir Dag Wollebæk, Bernard Enjolras, Kari Steen-Johnsen and Guro Ødegård, « After Utøya: How a High-Trust Society Reacts to Terror-Trust and Civic Engagement in the Aftermath of July 22 ", PS: Political Science and Politics, vol. 45, $\mathrm{n}^{\circ} 1,2012$, p. 32-37. Accessible à https://www.jstor.org/stable/41412718? seq=1\#metadata_info_tab_contents. Consulté le 23 avril 2020.

${ }^{7}$ Voir Charlotte Hath-Kelly, « Memory Wound », op. cit. Sur le vécu des parents, voir aussi l'étude sur le rôle des média par Kari Dygerov, Pål Kristensen et Atle Dyregrov, « Etterlattes opplevelser av medienes rapportering etter Utøya-terroren », Norsk medietidsskrift, vol. 23, $\mathrm{n}^{\circ} 3,2016$, https://www.idunn.no/nmt/2016/03/

etterlattes_opplevelser_av_medienes_rapportering_etter_utoey, https://doi.org/10.18261/issn. 0805-9535-2016-03-03. Consulté le 23 avril 2020.

7. Voir Charlotte Hath-Kelly, " Memory Wound ", op. cit. Sur le vécu des parents, voir aussi l'étude sur le rôle des média par Kari Dygerov, Pål Kristensen et Atle Dyregrov, «Etterlattes opplevelser av medienes rapportering etter Utøyaterroren ", Norsk medietidsskrift, vol. 23, n 3, 2016, https://www.idunn.no/nmt/2016/03/ etterlattes_opplevelser_av_medienes_rapportering_etter_utoey, https://doi.org/10.18261/issn. 0805-9535-2016-03-03. Consulté le 23 avril 2020.

8. Voir Franck Orban, «Avant et après le Vingt-deux Juillet : quatre décennies de menace terroriste en Norvège ", Revue d'histoire nordique, $\mathrm{n}^{\circ}$ 18, 2014, p. 199-233.

9. Hannah Arendt, Qu'est-ce que la politique ?, trad. Carole Widmaier et al., Paris, Seuil, coll. «Points », 2014, p. 170.

10. Frédérique Harry, " Discourses on Religion and Identity in Norway: Right-Wing Radicalism and AntiImmigration Parties ", dans Ergan Toğuşlu et al. (éds), New Multicultural Identities in Europe, Leuven, Leuven University Press, 2014, p. 161-170. 


\section{AUTEURS}

HARRI VEIVO

EA 4254 ERLIS, département d'études nordiques, université de Caen Normandie 